\section{$\$$ Research Square}

Preprints are preliminary reports that have not undergone peer review. They should not be considered conclusive, used to inform clinical practice, or referenced by the media as validated information.

\title{
A web-based adapted physical activity program (e-APA) versus health education program (e-HE) in patients with schizophrenia and healthy volunteers: study protocol for a randomized controlled trial (PEPSY V@Si)
}

\author{
Maxime Tréhout \\ Centre Hospitalier Universitaire de Caen \\ Elise Leroux \\ Universite de Caen Normandie \\ Lucile Bigot \\ Vasi \\ Solenne Jego \\ Universite de Caen Normandie \\ Pascal Leconte \\ Universite de Caen Normandie

\section{Emmanuel Reboursière} \\ Centre Hospitalier Universitaire de Caen

\section{Rémy Morello} \\ Centre Hospitalier Universitaire de Caen \\ Pierre-Alexandre Chapon \\ bodycap \\ Aline Herbinet \\ Vasi \\ Gaëlle Quarck \\ Universite de Caen Normandie \\ Sonia Dollfus ( $\boldsymbol{\nabla}$ dollfus-s@chu-caen.fr,dollfus@cyceron.fr ) \\ Universite de Caen Normandie https://orcid.org/0000-0002-6051-1748
}

\section{Study protocol}

Keywords: Schizophrenia; Adapted physical activity; Cerebral plasticity; Hippocampus; Cognition; Web; Health education;

Spectroscopy; MRI; DTI

Posted Date: March 19th, 2019

DOI: https://doi.org/10.21203/rs.2.456/v1

License: @ (i) This work is licensed under a Creative Commons Attribution 4.0 International License. Read Full License

Version of Record: A version of this preprint was published at European Archives of Psychiatry and Clinical Neuroscience on May 26th, 2020. See the published version at https://doi.org/10.1007/s00406-020-01140-z. 


\section{Abstract}

Background Patients with schizophrenia (SZ) present a higher level of cardiovascular morbidity than the general population due to poor physical fitness and a sedentary lifestyle. Moreover, despite major therapeutic advances in the management of SZ, some clinical symptoms remain resistant to pharmacological approaches. A large number of studies support the effectiveness of physical activity (PA) for both physical and mental health in SZ. The main objective of the present study is to assess the effects of a remote, web-based adapted PA program (e-APA) compared to a health education program (e-HE) on brain plasticity in SZ and healthy volunteers (HV). The secondary objectives are to assess the effects of e-APA on other cerebral variables as well as clinical, neurocognitive, circadian, biological, and physiological variables. Methods: The study is an interventional, multicenter, randomized open-label controlled trial. Forty-two SZ will be randomized to either the active group (e-APA, $\mathrm{N}=21$ ) or non-active group (e-HE, $\mathrm{N}=21$ ), and $42 \mathrm{HV}$ will be matched to $\mathrm{SZ}$ according to age, gender, and level of PA. Interventions will consist of 32 sessions (2x60 minutes/week, for 16 weeks) via supervised home-based videoconferencing. The active group will carry out an individualized PA program of moderate to vigorous intensity. Pre-and post-intervention cerebral magnetic resonance imaging (MRI) will be performed to evaluate changes in global hippocampal volume. Other cerebral assessments using diffusion tensor imaging, arterial spin labelling, spectroscopy and resting-state functional MRI will be performed. In addition, clinical, neurocognitive and circadian rhythms assessments as well as physical tests and biological analyses will be conducted. Discussion: To our knowledge, this is the first study aiming to evaluate the efficacy of APA delivered by supervised home-based videoconferencing in SZ. Moreover, using multimodal MRI, this study could clarify the pathophysiological mechanisms underlying the efficacy of APA. Finally, this innovative approach (remote, web-based APA) might also increase participation in long-term PA since PA-based programs are known to have low adherence and early dropout. The results of this study should promote the inclusion of PA interventions as a novel adjunctive therapy for SZ. Trial registration: ClinicalTrials.gov identifier: NCT03261817. Registered on 16 August 2017.

\section{Background}

Schizophrenia is a chronic mental disorder that affects approximately $1 \%$ of the world's population and that mainly manifests by positive symptoms (delusions and hallucinations), negative symptoms (e.g., blunted affect, alogia, asociality, avolition and anhedonia), disorganized behaviour and thought, and cognitive impairments (e.g., difficulties in working memory, episodic memory, executive functioning, attention and social cognition). These symptoms have a substantial impact on quality of life and well-being [1, 2]. Cognitive deficits and negative symptoms affect 50 to $80 \%$ of patients with schizophrenia (SZ) [3] and are usually present simultaneously $[4,5]$. They are associated with poor functional outcomes and cause disability in daily living, thus representing a serious mental health concern and an important target for interventions [6-9].

While antipsychotics are generally effective in improving positive symptoms, reducing the severity of the disease and preventing relapse [10], approximately 20 to $35 \%$ of SZ show a partial response or no response to standard antipsychotics, indicating treatment-resistant schizophrenia $[11,12]$. Moreover, available treatments offer only limited to minimal benefits on negative symptoms and cognitive deficits [13-15]. Recent meta-analyses did not find cognitive enhancement or negative symptoms improvement using adjunctive pharmacological treatment added to antipsychotic therapies [16, 17]. This supports the notion that non-pharmacological interventions like physical exercise should be recommended.

Moreover, SZ have dramatically higher levels of cardiovascular morbidity compared to the general population due to poor physical fitness (e.g., high rates of smoking and alcohol use disorders, poor diet, overweight and obesity), sedentary lifestyle (e.g., low levels of physical activity), antipsychotic side effects and low neurometabolic tolerance (e.g., overweight and obesity, glucose and lipid dysregulation, metabolic syndrome) [18-20]. SZ also have a reduction in life expectancy of 10 to 20 years $[21,22]$ and a significant excess mortality being two to three times more than that in the general population, which is largely due to increased cardiovascular diseases. Many of these physical health issues are related to modifiable risk factors that can be attenuated through lifestyle changes, including health education (HE) and physical activity (PA) programs. 
Emerging evidence from both animal and human studies suggests that PA is relevant as an adjuvant therapy in SZ in improving both physical and mental health as well as the functional outcomes [23]. Indeed, a number of important and recent meta-analyses have shown that PA significantly improved positive and negative symptoms, cognitive deficits and social functioning in this population [24-27]. PA also improved cardiovascular and respiratory capacities in SZ [28-31], by reducing overweight and obesity, insulin resistance, lipidic dysregulation, type II diabetes and metabolic syndrome [32-34]. Moreover, PA had a positive impact on sleep quality in SZ $[35,36]$. According to these studies, the clinical benefits of PA were underpinned by cerebral and biological mechanisms that stimulated cerebral plasticity and notably increased hippocampal volume through brain-derived neurotrophic factor (BDNF) [37, 38]. Nonetheless, further studies are required to better determine these pathophysiological processes. Despite these obvious benefits, PA can be harmful if it is not adapted to the patient's physiological capacities, particularly regarding to the cardiological risk. Indeed, it has been reported that antipsychotics could increase the risk of ventricular arrhythmia, sudden cardiac death, and electrocardiogram QT interval prolongation [39]. Therefore, PA programs should offer patient-adapted exercises and should be supervised by qualified supervisors [40]. It was partly in this context that "adapted" PA (APA) was developed in the 1970s. APA refers to movement, physical activity and sports in which special emphasis is placed on the adaptation of exercises to the capabilities and needs of individuals with limiting conditions, such as disabled, health-impaired, or aged people [41]. Thus, from a clinical approach, APA has been recently considered as an adjuvant therapy, and has developed novel therapeutic devices for populations with health concerns to improve their physical condition, health and quality of life.

Despite these evidences, most SZ remain physically inactive $[42,43]$. Indeed, a recent meta-analysis of 35 studies involving 3453 patients showed that $43.4 \%$ of SZ did not reach the 150 minutes per week of moderate to intensive PA recommended by the World Health Organization [42]. In addition, they spend an average of 11 hours a day in sedentary behaviours. To decrease the sedentary behaviour and its consequences on physical health, recommendations have been made and consist of 150 minutes per week of PA at moderate intensity, which is the equivalent of approximately 30 minutes of moderate-intensity exercises, 5 days per week, or 75 minutes per week of vigorous-intensity aerobic activity [44]. However, the implementation of PA-based programs is often hampered by poor adherence and early dropout in SZ [45]. Indeed, in randomized controlled trials including SZ, more than one participant in four dropped out PA programs (26.7\%; $95 \% \mathrm{Cl}=19.7 \%-35.0 \%)$. For this reason, and because clinical-based programs present many logistical barriers, it is often not feasible or cost-effective for health care professionals to administer PA programs over the long term. Evidence suggests that the more frequent barriers to engage in regular PA include the following: disinterest and poor motivation; feeling of sadness, anxiety and stress about PA in public, including social anxiety; tiredness; low physical self-perception and low self-efficacy; lack of social support to PA and stigma; comorbid physical health issues; poor access to facilities and cost; overweight and obesity, physical complaints, and low physical fitness; and side effects of medication [44, 46, 47]. Sustainable approaches to increasing participation in long-term PA are crucial. Therefore, studies suggest that PA programs delivered by a qualified professional and/or supervised throughout the duration of the intervention appear to be associated with significantly lower dropout rates [45]. Moreover, electronic health (e-health) technology has been developed in the general population to facilitate remote interactions and to encourage people to participate in PA over the long term [48-50]. The "e-intervention" term refers to any intervention delivered, carried out or received via electronic means. E-interventions can be advantageous as they tend to improve access, cost, convenience, education, care quality and effectiveness and can be delivered across an array of personal devices. In addition, einterventions enable participants to approach the exercises at their own pace and to overcome barriers associated with frequent practice without geographical limits. However, few studies using e-technologies have been conducted in SZ [51-56]. In part, this is due to a dearth of research on creating design models to make these technologies usable by SZ and others with cognitive impairments [57]. A lack of appropriate design models is a primary cause of poor designs of e-health applications for patients with cognitive impairments [58] and will continue to limit the inclusion and development of applications for SZ. Telepsychiatric approaches using videoconferencing technology could be an alternative tool to bypass these limitations [59]. To our knowledge, no study has investigated the impact of APA programs delivered, supervised and monitored via web-based videoconferencing in SZ.

The purpose of the present study is to describe the protocol of an interventional randomized controlled trial (PEPSY V@Si). Its aim is to demonstrate in SZ that an APA program delivered via web-based videoconferencing (e-APA, active group) improves

Page $3 / 21$ 
brain plasticity assessed by the hippocampal volumes when compared to an health education program (HE, non-active control group) using the same technologies in the same conditions (e-HE). Each group of patients is compared with an healthy volunteers (HV) group involved in the same processes. The secondary objective is to assess the effects of e-APA on other cerebral variables as well as clinical, neurocognitive, biological, and physiological variables and circadian rhythms.

\section{Methods}

\section{Study design and objectives}

PEPSY V@Si is designed as an interventional, multicenter, randomized controlled open-label trial. The main objective is to demonstrate an increase in overall hippocampal volumes (right and left) in SZ receiving a 16-week, web-based e-APA program compared to SZ receiving a 16-week, web-based e-HE program (Figure 1). SZ are randomized to either the active group (e-APA) or the non-active control group (e-HE). Subsequently, HV are matched to SZ on age, gender, and baseline level of PA. Both HV receive the same interventions as the corresponding SZ. The present protocol followed the Standard Protocol Items: Recommendations for Interventional Trials (SPIRIT) guidelines and fulfilled the SPIRIT checklist (Additional file 1).

All participants ( $\mathrm{SZ}$ and $\mathrm{HV}$ ) are assessed for the primary endpoint at the start (baseline assessment, $\mathrm{V} 1$ ) and at the end of the intervention (the 16-week follow-up assessment, V6). Other visits are conducted between V1 and V6 to assess secondary endpoints.

\section{Ethics and regulatory considerations}

Written informed consent is obtained from all participants before their inclusion. The protocol was approved by Health Authorities (ANSM; Agence Nationale de Sécurité du Médicament et des produits de santé) on 19 July 2016 (ID-RCB number 2016-A00930-51) and by the ethical committee (Comité de Protection des Personnes, CPP Nord-Ouest IV, France) on 10 January 2017 (CCP16/39) in compliance with French regulations. Trial registration was carried out at ClinicalTrial.gov on 25 August 2017 (NCT03261817).

\section{Recruitment and randomization}

Forty-two SZ are recruited from Caen and Rouen University Hospitals (France). SZ are diagnosed according to the Diagnostic and Statistical Manual of Mental Disorders (DSM-IV-TR, $4^{\text {th }}$ edition, text revision) criteria with the structured Mini-International Neuropsychiatric Interview (MINI, French version 5.0.0). All patients are stabilized outpatients with no change in their treatment over the last two months prior to their inclusion.

Forty-two HV are recruited from the general community in Caen via an announcement broadcast by press, posting or mailing. Matching criteria are checked by phone and supplementary interviews.

For both groups, the clinicians assess the subject's eligibility and provide to each subject and to the patient's legal representative, comprehensive, verbal and written information regarding the objectives and procedures of the study as well as the possible risks.

A signed informed consent is obtained from each participant and/or legal representative for SZ prior to undertaking any studyrelated procedure. SZ patient who does not wish to take part in the study continues to have treatment as usual.

Inclusion and exclusion criteria are presented in Table 1 for SZ and in Table 2 for HV. 
Randomization is established only for eligible patients and is performed in a 1:1 ratio by a central computer-assisted procedure centralized at the PEPSY V@Si data center (Ennov Clinical Software ${ }^{\circledR}$ ). SZ are assigned either to the active group (eAPA) or to the control group (e-HE). HV are recruited and matched to SZ on age, gender and physical activity level according to the Ricci and Gagnon self-questionnaire [60]. Each participant who enters into the study receives a unique identification number.

\section{Intervention / study protocol}

In this study, each intervention delivered, carried out or received electronically is called an "e-intervention". Both interventional activities, e-APA and e-HE, are delivered via videoconferencing using a secure web-based platform named SAPATIC ${ }^{\circledR}$ (Santé Activités Physiques Adaptées utilisant les Technologies de l'Information et de la Communication). All participants' data are hosted on a HADS server (Hébergeur Agrée de Données de Santé). All participants are able to connect to the platform at home or in a connected room in ambulatory care services. The time spent with the intervention staff are the same in both groups, and a specific time schedule are followed to ensure that each session lasts 60 minutes. Participants receive 2 sessions per week for 16 weeks, which is a total of 32 sessions.

The intervention in both groups begins with a motivational interview for increasing e-APA or e-HE participation. In the e-APA group, the motivational interview allows the professional to better understand the expectations of participants in terms of PA.

SZ assigned to the active or to the control group receive their usual medications during the 16-week program.

\section{Active group}

For all participants (SZ and HV) assigned to the active group, e-APA sessions is implemented in addition to daily life activities.

The e-APA program, designed according to the American College of Sports Medicine (ACSM) guidelines [61], is individually supervised by qualified APA professional trainers from the V@Si company selected from a list of certified professionals chosen for the study. Physical exercises are individualized and adapted for each participant according to standardized guidelines and to his/her sporting past, treatment, preferences and fitness level (estimated by the Global Physical Activity Questionnaire - GPAQ) [62], baseline maximal exercise test and maximal strength test. Exercises are conducted at a moderate to vigorous intensity (60 to $75 \%$ of maximal heart rate based on participant's baseline maximal exercise test), assessed through real-time heart rate monitoring using cardiac sensors (Zephyr BioHarness ${ }^{\circledR}$ or OnRythm $500 \AA$ ). Exercises are performed with an intensity that allows the participant to speak with moderate breathlessness.

Three alternative types of exercises are proposed to participants: 1) aerobic exercises, 2) resistance exercises to maintain or increase arm and leg muscle strength, and 3) balance exercises combining flexibility and coordination. The number of repetitions and the difficulty of the exercise are gradually increased during the sessions and are modulated according to the participant's capacities at the time of the exercises. Difficulties of activities are increased in duration, then in frequency, and ultimately in intensity only if the participant tolerates previous increases. The heart rate, the perceived exertion as assessed by the Borg Rating of Perceived Exertion Scale [63], and the perceived dyspnea (visual analogue scale) evaluated at each session are the three indicators that allow the professional to ensure that work is done at the targeted intensity while respecting the participant's tolerance.

Each 60-minute APA session is composed of a verbal contact sequence, warm-up exercises (5 to 10 minutes), aerobic, resistance or balance exercises (40 minutes), a cool-down period (5 to 10 minutes) and finally a verbal feedback sequence. The warm-up and recuperation periods and verbal sequences are account for at least $30 \%$ of the session duration. During the e-APA program, participants are asked to report and estimate, in a dedicated activity digital booklet on the SAPATIC platform, 
their dyspnea (on a visual analogue scale) as well as their perception of the effort and muscle pain in the pre- and postsession periods. This booklet can be used to help participants recall exercises performed and difficulties encountered. Realtime videoconferencing plus heart rate monitoring allow the professional to assess the participant's tolerance and difficulties in completing the targeted PA level during aerobic training, resistance exercises, and balance exercises.

\section{Control group}

The participants (SZ and HV) assigned to the control group receive an e-HE program including web-delivered information on the main mental illnesses, the benefits of physical activity, healthy lifestyles (dietary balance, sleep cycle, stress management), alcohol, drug, tobacco and cardiovascular risk factors and are conducted with the same conditions (duration and frequency) as the active group. Individual training of participants in the e-HE group is carried out by researchers (EL, LB, MT, SG).

The 50-minute training is followed by a 10-minute quiz aiming to assess their involvement in the course.

\section{Specific aims}

The main objective of this study is to demonstrate that e-APA compared to e-HE can improve cerebral plasticity reflected by an increase in the overall hippocampal volumes in both the SZ and HV groups.

The secondary objective is to assess the impact of the active intervention (e-APA) compared to the non-active intervention (e$\mathrm{HE}$ ) on the following other variables in both SZ and HV:

1. Cerebral variables (volumetric changes in the different subregions of the hippocampus (cornu ammonis (CA: CA1, CA2-34), subiculum and dentate gyrus); cerebral cortical thickness; N-acetyl aspartate (NAA) reflecting neuronal integrity in the hippocampus; integrity of white matter in whole brain and in the frontomedio-hippocampal fibres; and cerebral blood irrigation)

2. Physiological variables (i.e., neuromuscular, cardiovascular (heart rate variability) and aerobic)

3. Cognitive variables (i.e., working memory, episodic memory, attentional and executive functions)

4. Circadian rhythms (i.e., temperature, motor activity (actimetry) and wake-sleep cycle)

5. Clinical status of participants (i.e., severity of symptoms only for SZ, quality of life, level of activity and physical abilities)

6. Biological variables (i.e., fasting glucose and lipidic dysregulation)

\section{Assessments}

All participants are assessed at 2 time points for the primary endpoint (V1 and V6) and at 6 time points for the secondary endpoints (from V1 to V6). The first assessment occurs at baseline (V1) prior to randomization. The final assessment takes place after completion of the intervention (V6). Other visits occur during the intervention (V2, V3, V4, V5). The content of all assessments (V1, V2, V3, V4, V5 and V6) is outlined in Table 3. 
The primary outcome is the change in left and right hippocampal volumes between V1 and V6. Neuroimaging data is acquired on a 3 Tesla scanner (Intera Achieva 3T Quasar Dual, Philips Medical System, Netherlands) at V1 and V6. A proton density-weighted $\mathrm{T}_{2}$ image focalized on the hippocampus is acquired using anatomical MRI (aMRI).

\section{Secondary endpoints}

The secondary outcomes consist in assessing neuroimaging data, neurocognitive, clinical, biological, cardiorespiratory function, body composition and muscle strength, motor activity and circadian rhythms, psychoactive substance use, and psychosocial functioning between different visits (V1 to V6) (see Table 3).

\section{Neuroimaging}

Volumetric changes in the different subregions of the hippocampus are assessed from proton density-weighted $T_{2}$ images; cerebral cortical thickness will be assessed from $\mathrm{T}_{1}$-weighted images using aMRI; NAA in the hippocampi is assessed using the magnetic resonance spectroscopy; integrity of white matter is assessed through diffusion parameters (fractional anisotropy, radial diffusivity and mean diffusivity reflecting the number of axons and the degree of myelination of fibres) using diffusion tensor imaging; the functional resting state is assessed with functional MRl; and cerebral blood irrigation is assessed through arterial spin labelling imaging.

\section{Neurocognitive tests}

Episodic and/or working memories are assessed using the Wechsler Adult Intelligence Scale (WAIS) Memory subtest [64, 65], the Rey Auditory Verbal Learning Test (RAVLT) [66] and the Corsi test [65]; attentional functions are assessed using the Stroop test [67] and the WAIS Coding subtest; executive functions are assessed using the Trail Making Test (TMT) [68], the d2 test [69] and the verbal fluency [70].

\section{Clinical symptoms}

Psychotic and affective symptoms are assessed using the Positive and Negative Syndrome Scale (PANSS) [71], SelfAssessment of Negative Symptoms (SNS) [72], Brief Negative Syndrome Scale (BNSS) [73], and Hospital Anxiety and Depression Scale (HADS) [74]. General symptoms are appraised using the Clinical Global Impression scale (CGI) [75]. Insight of illness is evaluated with the Insight Scale (IS) [76].

\section{Biological data}

Fasting blood exams are performed in order to measure the level of glucose, triglycerides, total cholesterol, high-density lipoprotein cholesterol (HDLc), and low-density lipoprotein cholesterol (LDLC).

\section{Cardiorespiratory fitness}


Maximal oxygen uptake $\left(\mathrm{V}_{2} \mathrm{max}\right)$ are measured at $\mathrm{V} 1$ and $\mathrm{V} 6$ during a maximal exercise test on an electromagnetic bicycle ergometer (Ergoline er $900^{\circledR}$ ). The exercise test is triangular by direct measurement. Participants have to pedal as long as possible while the load is increasing by 20 watts every 2 minutes. Developed power, $\mathrm{O}_{2}$ and $\mathrm{CO}_{2}$ flow rates are recorded before the beginning of the test (resting values), at each level (relative values), until the end of the test (maximal values) and during the following 3 minutes of passive recovery (recovery values). Heart rate and electrocardiogram are also recorded continuously, and blood pressure is recorded at the end of each level. The ventilatory regime and gas exchange are measured cycle to cycle continuously with a pneumotachograph. Parameters such as ventilation, oxygen consumption and respiratory quotient are recorded.

\section{Body composition and muscle strength}

Body composition assessment is performed using the BIA $101^{\circledR}$ impedance analyser, which measures body impedance using four electrodes (two on the right wrist and two on the right ankle). The patient is lying down to avoid any pressure variation that would affect the accuracy and reproducibility of the measurements.

Evaluation of maximal muscle strength is recorded with a hand dynamometer (model TK- $200^{\circledR}$ ). The force developed in isometry by the upper limbs (the gripping force of the hand) is analysed. Three successive measurements are collected in a standardized position for each arm, consisting of an orthostatic position with their arm at the side and the instrument held in the extension of the forearm. A recovery of one minute is granted between each repetition. Participants need to maintain their effort for 6 seconds to record maximal muscle tension.

\section{Motor activity and circadian rhythms}

Actimetry recording is performed continuously for 7 days at baseline (V1) and after the intervention (V6) using a lightweight, autonomous and waterproof actimeter (e-tact actimeterâ, BodyCap, France). The following parameters are analysed with ACTISOMM ${ }^{\circledR}$ software [77]: duration of the total night rest period; duration of the total period of daytime activity; average night activity; average daytime activity; magnitude, mesor and acrophase of circadian rhythms of motor activity; and night inactivity index (\%) (corresponding to the number of minutes where the amount of movement is zero, divided by the number of minutes of the night). Coupled with the sleep schedule, the data allow us to analyse the sleep/wake cycle.

The GPAQ [62] is completed by participants. It collects information on the practice of PA in the following three situations (i.e., activities at work, moving from place to place, and recreational activities) and on sedentary behaviours. This questionnaire is used as part of the program start interview, because it provides a diagnostic evaluation of the participant's habits, allowing the APA program to be individualized.

Moreover, central temperature recording is performed via the gastrointestinal tract using the e-Celsius ${ }^{\circledR}$ performance telemetry system (BodyCap, France) before and after the intervention program. The temperature is recording from ingestion until faecal elimination of the capsule. This device consists of a portable monitor that allows the continuous collection and recording internal temperature data. This electronic capsule, made in a biocompatible PVC (polycarbonate) envelope, consists of a temperature sensor that measures the gastrointestinal temperature every 30 seconds. The temperature data are then transmitted by telemetry to the monitor which records them in real time.

\section{Psychoactive substance use}


The severity of alcohol use is appraised with the Alcohol Use Disorders Identification Test (AUDIT) [78]. This tool is a 10item screening questionnaire with 3 subscales: the first scale includes 3 questions on the amount and frequency of drinking; the second scale includes 3 questions on alcohol dependence; and the third scale includes 4 questions on problems caused by alcohol. Low-risk consumption is defined by an AUDIT score below 6 for women or 7 for men; a score between 6 for women or 7 for men and 12 determines excessive alcohol consumption, whereas a score greater than or equal to 13 in either gender suggests alcohol dependence.

Participants are also asked about current or past tobacco consumption, their desire to quit tobacco and their degree of dependence according to the Fagerström test for Nicotine Dependence [79]. Little or no dependence was defined by a score of 0 or 1 , moderate dependence by a score of 2 or 3 , and severe dependence by a score above 3 .

The use of cannabis is assessed by the Cannabis Abuse Screening Test (CAST) [80]. It is a 6-item scale designed for adolescents and young adults to identify problematic forms of cannabis use that might lead to negative social and health consequences. Scores can vary from 0 to 24, and users are defined without risk when they have a score below 3 , with a low risk for a score equal to 3 and below 7 or with a high risk of dependence for a score greater than or equal to 7 .

\section{Psychosocial functioning}

The Social Adjustment Scale Self-Report (SAS-SR) [81] provides an understanding of an individual's level of satisfaction with his or her social situation.

Ryff's Psychological Well-Being Scale (PWB) [82] evaluates the participant's psychological well-being through six dimensions (autonomy, environmental mastery, personal growth, positive relations with others, purpose in life, and self-acceptance).

\section{Sample size calculations}

The number of participants to be included takes into account the risk of participants being lost to follow-up. Based on preliminary and comparable studies (12-week PA program in SZ), one could estimate that $20 \%$ of participants would discontinue the protocol prematurely [83].

Given the literature regarding the impact of PA on hippocampal volume in SZ [84], a difference of $6 \%$ between mean volume changes (as a percentage of total brain volume) between groups with and without PA for a standard deviation of hippocampal volume(s) of $8 \%$ is quite conceivable and clinically relevant based on Pajonk's study [84]. The standard deviation (Sv) of the change in hippocampal volume(s) is estimated at $5 \%$ based on a correlation coefficient ( $r$ ) between baseline assessments (V1) and at the end of PA program at least equal to 0.8.

For an alpha risk of $5 \%$ and a power of $80 \%$, the number of participants to be included per group is 12 . Given the small number of participants and a distribution of changes in relative hippocampal volumes possibly not being normal, a Mann-Whitney test can be used. In this case, a correction must be made using the ARE (asymptotic relative efficiency) of the Mann-Whitney test in relation to the Student's test, which amounts to the following ratio: 12 / $0.864=14$, i.e., the number of participants to be included per group. While it is customary to consider a $20 \%$ increase for those lost to follow-up, this brings the number of participants to 17 per group. Our inclusion capacity is higher, and to meet the tests that will be carried out for the secondary objectives, 84 participants are included ( 21 for each of the 4 groups).

\section{Statistical analysis}


In order to meet the main objective, Student's t-test will be used to determine if the averages of two samples are significantly different (SZ_e-APA vs SZ_e-HE). In a first step, to analyze primary and secondary outcomes, analyses of covariance (ANCOVAs) will be computed between the four groups of participant (SZ_e-APA vs. SZ_e-HE vs. HV_e-APA vs. HV_e$\mathrm{HE}$ ). Primary or secondary outcomes will be considered as the dependent variable and the group as the independent variable. In a second step, gender and age will be included as covariates in the analyses. Post-hoc analyses will be conducted when a significant main effect is observed using a Tukey's HSD test to correct for multiple comparisons. We will also conduct intragroup Pearson correlation analyses in the SZ groups to evaluate the relationships between neuroimaging, physiological data with clinical, neurocognitive data and medication. The same correlations will be tested in the HV group except with medication. The correlations will be considered statistically significant after the adjustment for multiple comparisons. All statistical analyses will be performed with JMP v12.0 Software (SAS Institute, Inc., Cary, NC) or IBM ${ }^{\circledR}$-SPSS ${ }^{\circledR} 25.0$ (IBM Corp. Released 2017. IBM SPSS Statistics for Windows, Version 25.0. Armonk, NY: IBM Corp). The significance level is set at $P \square$ 0.05 .

Study outings before the start or the beginning of interventions (e-APA or e-HE) before the $10^{\text {th }}$ session will be systematically replaced. Study outings after the $10^{\text {th }}$ session will be not replaced. Any participant who leaves prematurely program after the $10^{\text {th }}$ session and before the end of the study will benefit for all the examinations planned at the end of the study. Causes of nonobservances will be analyzed and listed. Rates and reasons for premature study outing will be analyzed in relation to their impact on the obtained results. In case of premature outing, data will be collected in the CRF at the time of the event and will be taken into account in the analysis of the primary outcome (hippocampal volumes) if the number of planned sessions exceeds $75 \%$ (24 sessions or more). Analysis will be conducted in intention to treat and will concern all of the variables. Noncompliant participants will be defined as the absence to 4 consecutive sessions.

\section{Data management and monitoring}

Data are initially collected and anonymously registered using paper forms (CRF), which are then securely stored and extracted into protected electronic files (e-CRF) in Ennov Clinical Software ${ }^{\circledR}$.

The medical procedures used in this trial comply with the most recent recommendations of the Declaration of Helsinki and French Public Health Law 2004-806 of 9 August 2004 on participant protection and safety in accordance with good clinical practice. A data manager mandated by the trial sponsor (CHU de Caen, Avenue de la Côte de Nacre, CS30001, Caen, France) ensures monitoring of this trial to guarantee that accurate, full, and reliable data are collected.

At the end of the study, the data review committee, comprising the data manager and the biostatistician will review all deviations from the protocol. The committee will qualify deviations as major or minor and shall clarify the relevance of the data with respect to these deviations: conservation of the data (for minor deviation) or exclusion of the data (for major deviation).

\section{Safety and emergency procedures}

All adverse events (AEs) reported by the participants or observed by the clinicians and the APA professionals are recorded. All serious AEs are reported to the ANSM.

The clinical investigator is responsible for the record of all the relevant information regarding any event occurring during the eAPA or e-HE program. The investigator is requested to assess the relationship between the intervention and occurrence of each AE using clinical criteria. Alternative causes, such as natural history of the underlying diseases, concomitant therapy, other risk factors, and temporal relationship of an event to the intervention, are considered and investigated. 
The investigators and all other appropriate persons are informed of events that could adversely affect the safety of participants in a timely manner during the study duration.

Participants are able to interrupt their participation in the study if they wish, at any time and for whatever reason, or according to the decision of the investigator. Reasons for premature study exit can include a relapse requiring hospitalization or a significant increase in medications, a refusal by the participant to continue, a withdrawal of consent, a protocol violation requiring a study exit, a serious $\mathrm{AE}$, a decision of the investigator, and a decision of the funding agency or French Health authorities.

\section{Dissemination policy and data sharing}

The University Hospital of Caen will keep participants informed of the overall results at the end of the study. All publications must comply with the rules set out in the Consortium Agreement and the International Committee of Medical Journal Editors (ICMJE). In case of additional studies, the results can only be published with the agreement of the principal investigator and the statistician, and only after publication of the main study to be quoted. Analysis of the results will be the object of communications in congresses and publications. The text of publications and papers will be discussed with all investigators participating in the trial.

\section{Discussion}

Through this study, we aim to demonstrate the benefits of a remote, web-based APA videoconferencing program (e-APA) in patients with schizophrenia; this kind of APA program has never been investigated in SZ. The trial consists of an interventional randomized controlled study designed to examine whether a 16-week APA program delivered via web-based videoconferencing (e-APA) improves cerebral, clinical, neurocognitive, biological and physiological variables in SZ compared to a non-active controlled group (e-HE) also delivered via web-based videoconferencing. We hypothesize that the e-APA program may have benefits on hippocampal plasticity but also on schizophrenia-related symptoms.

Considered as an adjunctive treatment in curative and supportive interventions, APA is an original and promising nonpharmaceutical strategy that may help to better manage symptoms and health concerns in SZ [44]. APA is cost effective, feasible and efficient in various symptomatic features and in cardiovascular prevention of vulnerable and sedentary populations such as SZ. For patients with severe mental illness, such as SZ, adherence to treatment and PA recommendations are often poor, limiting their recovery, functional outcomes and the improvement of their general health $[45,85]$. Using etechnologies may bypass many barriers to PA practice in this population and enhance the engagement and participation of patients in PA programs in clinical practice [86-88].

There are several originalities in this study. First, regarding the methodology of the randomized controlled trial, we used a nonactive control group (e-HE) to explore whether the active intervention could improve brain plasticity, symptoms and physical concerns more than the non-active intervention. Exploring the brain with multimodal MRI could allow us to best identify how APA impacts the brain by assessing structural (grey and white matter), functional and anatomical connectivity, and metabolic and arterial flow of the brain. Moreover, comparing SZ and HV will allow us to determine if the expected benefits on brain plasticity and other variables occur in both groups or if the effects of PA in SZ are attenuated, in line with previous literature but not entirely elucidated yet $[89,90]$. Second, both interventions are delivered by qualified professionals in real time using interactive videoconferencing, reproducing face-to-face interventions and allowing participants and professionals to see each other with an aim to reinforce the participants' motivation. Videoconferencing allows everyone to see themselves through the screen and instantaneous correct the movements if they are badly executed, and finally, it allows the professional to interact and reassure the patient to decrease the level of anxiety. The self-image brings about a reappropriation of one's body that is capable of doing (self-confidence). The use of e-technologies and videoconferencing may also help to facilitate participation in PA over the long term. Moreover, it will enable participants to approach the intervention at their own pace and to overcome 
barriers towards regular PA without geographical limits. Third, the e-APA intervention will consist of adapted exercises that will simultaneously allow the participant to control the commitment to practice and provide constant metabolic expenditure measures (cardiac monitoring) to foster the motivation of the participants and adapt the program to the specific needs of each one, while standardizing the program. Fourth, many variables that have never been simultaneously explored before in SZ, such as multimodal imaging data along with clinical, cognitive, biological, physiological and circadian rhythms assessments, are investigated in this study. Finally, findings from this study will add to the growing scientific literature on the impact of PA in SZ. Furthermore, the study results may have clinical implications for the improvement of multimodal management of SZ and other severe mental illnesses.

\section{Abbreviations}

ACSM: American College of Sports Medicine; AE: adverse event; aMRI: anatomical magnetic resonance imaging; ANCOVAs: analyses of covariance; ANSM: Agence Nationale de Sécurité du Médicament; APA: adapted physical activity; ARE: asymptotic relative efficiency; AUDIT: Alcohol Use Disorders Identification Test; BDNF: brain-derived neurotrophic factor; BMI: body mass index; BNSS: Brief Negative Syndrome Scale; CAST: Cannabis Abuse Screening Test; CGI: Clinical Global Impression scale; CRF: case report form; DSM-IV-TR: Diagnostic and Statistical Manual of Mental Disorders, $4^{\text {th }}$ edition, text revision; DTI: diffusion tensor imaging; e-APA: web-based adapted physical activity program; e-HE; web-based health education program; GPAQ: Global Physical Activity Questionnaire; HADS: Hospital Anxiety and Depression Scale; HDLc: high-density lipoprotein cholesterol; HE: health education program; HV: healthy volunteers; IS: Insight Scale; LDLc: low-density lipoprotein cholesterol; MRI: magnetic resonance imaging; NAA: N-acetyl aspartate; PA: physical activity; PANSS: Positive and Negative Syndrome Scale; PWB: Ryff's Psychological Well-Being Scale; RAVLT: Rey Auditory Verbal Learning Test; SAPATIC: Santé Activités Physiques Adaptées utilisant les Technologies de l'Information et de la Communication; SAS-SR: Social Adjustment Scale Self-Report; SNS: Self-Assessment of Negative Symptoms; SZ: patients with schizophrenia; $\mathrm{VO}_{2}$ max: maximal oxygen uptake; WAIS: Wechsler Adult Intelligence Scale.

\section{Declarations}

\section{Trial status}

The current PEPSYV@SI protocol version is 5 dated 6 March 2018. The first participant was recruited in September 2017, and the study is currently enrolling participants. The study is expected to be completed in December 2021.

\section{Ethics approval and consent to participate}

The protocol was submitted to the French Health Authoritiy, that is, the Agence Nationale de Sécurité du Médicament et des produits de santé (ANSM) for formal approval of the study conduct to ensure that the study meets the local regulations and to a properly constituted Ethics Committee. The project was approved by Health Authorities (ANSM) on 19 July 2016 (ID-RCB number 2016-A00930-51) and by the local ethical committee (Comité de Protection des Personnes, CPP Nord-Ouest IV, France) on 10 January 2017 in compliance with French regulations. Trial registration was carried out at ClinicalTrial.gov on 25 August 2017 (NCT03261817). Initial information about the study is given to eligible participants by the regular staff during a routine appointment or by co-workers. Further detailed written and oral information about the trial are then given by the project coworker. It is assured that participation is voluntary, that the participants can withdraw at any time and receive ordinary treatment whether they choose to participate or not. Each participant provides written informed consent before any intervention is begun.

\section{Consent for publication}

Page $12 / 21$ 
Not applicable

\section{Availability of data and materials}

The datasets are available from the trial promotor on request (gaillard-c@chu-caen.fr).

\section{Competing interests}

MT participated in educational conferences for the following industrial laboratories: Otsuka, Lundbeck, and Janssen.

SD has been an expert and consultant or participated in educational conferences for the following industrial laboratories: Gedeon Richter, Lundbeck Otsuka, Roche, Takeda, Fabre, Janssen and ONO Pharma. She also has a license agreement on SNS with MedAvante-ProPhase.

EL, SJ, PL, ER, RM and GQ have no competing conflicts.

AH is manager of V@Si company and LB is employed by V@Si.

PA is employed by Bodycap compagny.

\section{Funding}

The PEPSY European project is co-funded by the Normandy County Council, European Union. This work was also supported by the Fondation Pierre Deniker. Funding sources were not involved in the study design, the collection, analysis and interpretation of data, the writing of the report, or the decision to submit the paper for publication.

\section{Authors' contributions}

MT: participation in HE program conception and dispensation, subject recruitment, participant assessments, MRI assessments, data analysis, and manuscript drafting and revision. EL: HE program conception and dispensation, subject prerecruitment, MRI assessments, imaging pre- and post-processing, data analysis, neurocognitive assessments, and manuscript revision. SJ: HE program conception and dispensation and neurocognitive assessments. PL: methods on evaluation of APA. LB: APA videoconferencing and SAPATIC platform management. AH: study conception and design, APA program conception, and SAPATIC platform conception and management. ER: exercise test dispensation. RM: methodological conception and manuscript revision. PAC: e-Celcius and Zephyr BioHarness ${ }^{\circledR}$ management. GQ: study conception and design, circadian data analysis, and manuscript revision. SD: study conception and design, principal investigator, patient recruitment, participant assessments, MRI assessments, data analysis, and manuscript revision.

All authors have read and approved the final manuscript.

\section{Acknowledgements}


We thank the DRCl (Délégation à la Recherche Clinique et à I'Innovation) and CRC (Centre de Recherche Clinique) staffs for their contribution to the study conception and for the promotion and management of the protocol proceedings. We also thank Pr Olivier Guillin from the University Hospital of Rouen and all associated investigators for their clinical contributions. We thank collaborators from GIP Cyceron, Nicolas Delcroix for MRI sequences, Frédéric Briend for MRI spectroscopy sequence and assessments, Mickael Naveau for the database, and Gaëlle Chetelat and Robin de Flores for hippocampal sequence. We thank Drs Perrine Brazo, Sophie Meunier-Cussac, Aurélie Montagne, Pierrick Lebain, Anaïs Vandevelde, for patient recruitment. Finally, we thank patients and healthy volunteers for their upcoming participation in the study.

\section{References}

1. van Os J, Kapur S. Schizophrenia. Lancet Lond Engl. 22 août 2009;374(9690):635-45.

2. Insel TR. Rethinking schizophrenia. Nature. 11 nov 2010;468(7321):187-93.

3. Palmer BW, Heaton RK, Paulsen JS, Kuck J, Braff D, Harris MJ, et al. Is it possible to be schizophrenic yet neuropsychologically normal? Neuropsychology. juill 1997;11(3):437-46.

4. Cohen AS, Saperstein AM, Gold JM, Kirkpatrick B, Carpenter WT, Buchanan RW. Neuropsychology of the deficit syndrome: new data and meta-analysis of findings to date. Schizophr Bull. sept 2007;33(5):1201-12.

5. Hartmann-Riemer MN, Hager OM, Kirschner M, Bischof M, Kluge A, Seifritz E, et al. The association of neurocognitive impairment with diminished expression and apathy in schizophrenia. Schizophr Res. déc 2015;169(1-3):427-32.

6. Bowie CR, Leung WW, Reichenberg A, McClure MM, Patterson TL, Heaton RK, et al. Predicting schizophrenia patients' realworld behavior with specific neuropsychological and functional capacity measures. Biol Psychiatry. 1 mars 2008;63(5):505-11.

7. Rabinowitz J, Levine SZ, Garibaldi G, Bugarski-Kirola D, Berardo CG, Kapur S. Negative symptoms have greater impact on functioning than positive symptoms in schizophrenia: analysis of CATIE data. Schizophr Res. mai 2012;137(1-3):147-50.

8. Fervaha G, Foussias G, Agid O, Remington G. Motivational and neurocognitive deficits are central to the prediction of longitudinal functional outcome in schizophrenia. Acta Psychiatr Scand. oct 2014;130(4):290-9.

9. Ventura J, Subotnik KL, Gitlin MJ, Gretchen-Doorly D, Ered A, Villa KF, et al. Negative symptoms and functioning during the first year after a recent onset of schizophrenia and 8 years later. Schizophr Res. févr 2015;161(2-3):407-13.

10. Leucht S, Tardy M, Komossa K, Heres S, Kissling W, Davis JM. Maintenance treatment with antipsychotic drugs for schizophrenia. Cochrane Database Syst Rev. 16 mai 2012;(5):CD008016.

11. Sheitman BB, Lieberman JA. The natural history and pathophysiology of treatment resistant schizophrenia. J Psychiatr Res. août 1998;32(3-4):143-50.

12. Lindenmayer JP. Treatment refractory schizophrenia. Psychiatr Q. 2000;71(4):373-84.

13. Lieberman JA, Stroup TS, McEvoy JP, Swartz MS, Rosenheck RA, Perkins DO, et al. Effectiveness of antipsychotic drugs in patients with chronic schizophrenia. N Engl J Med. 22 sept 2005;353(12):1209-23.

14. Nielsen RE, Levander S, Kjaersdam Telléus G, Jensen SOW, Østergaard Christensen T, Leucht S. Second-generation antipsychotic effect on cognition in patients with schizophrenia-a meta-analysis of randomized clinical trials. Acta Psychiatr Scand. mars 2015;131(3):185-96.

15. Veerman SRT, Schulte PFJ, de Haan L. Treatment for Negative Symptoms in Schizophrenia: A Comprehensive Review. Drugs. sept 2017;77(13):1423-59.

16. Choi K-H, Wykes T, Kurtz MM. Adjunctive pharmacotherapy for cognitive deficits in schizophrenia: meta-analytical investigation of efficacy. Br J Psychiatry J Ment Sci. sept 2013;203(3):172-8.

17. Correll CU, Rubio JM, Inczedy-Farkas G, Birnbaum ML, Kane JM, Leucht S. Efficacy of 42 Pharmacologic Cotreatment Strategies Added to Antipsychotic Monotherapy in Schizophrenia: Systematic Overview and Quality Appraisal of the Meta-analytic Evidence. JAMA Psychiatry. 17 mai 2017;

Page 14/21 
18. von Hausswolff-Juhlin Y, Bjartveit M, Lindström E, Jones P. Schizophrenia and physical health problems. Acta Psychiatr Scand Suppl. 2009;(438):15-21.

19. DE Hert M, Correll CU, Bobes J, Cetkovich-Bakmas M, Cohen D, Asai I, et al. Physical illness in patients with severe mental disorders. I. Prevalence, impact of medications and disparities in health care. World Psychiatry Off J World Psychiatr Assoc WPA. févr 2011;10(1):52-77.

20. Correll CU, Detraux J, De Lepeleire J, De Hert M. Effects of antipsychotics, antidepressants and mood stabilizers on risk for physical diseases in people with schizophrenia, depression and bipolar disorder. World Psychiatry Off J World Psychiatr Assoc WPA. juin 2015;14(2):119-36.

21. Laursen TM. Life expectancy among persons with schizophrenia or bipolar affective disorder. Schizophr Res. sept 2011;131(1-3):101-4.

22. Walker ER, McGee RE, Druss BG. Mortality in mental disorders and global disease burden implications: a systematic review and meta-analysis. JAMA Psychiatry. avr 2015;72(4):334-41.

23. Tréhout M, Dollfus S. [Physical activity in patients with schizophrenia: From neurobiology to clinical benefits]. L'Encephale. 5 juill 2018;

24. Rosenbaum S, Tiedemann A, Sherrington C, Curtis J, Ward PB. Physical activity interventions for people with mental illness: a systematic review and meta-analysis. J Clin Psychiatry. sept 2014;75(9):964-74.

25. Firth J, Cotter J, Elliott R, French P, Yung AR. A systematic review and meta-analysis of exercise interventions in schizophrenia patients. Psychol Med. mai 2015;45(7):1343-61.

26. Dauwan M, Begemann MJH, Heringa SM, Sommer IE. Exercise Improves Clinical Symptoms, Quality of Life, Global Functioning, and Depression in Schizophrenia: A Systematic Review and Meta-analysis. Schizophr Bull. mai 2016;42(3):588-99.

27. Firth J, Stubbs B, Rosenbaum S, Vancampfort D, Malchow B, Schuch F, et al. Aerobic Exercise Improves Cognitive Functioning in People With Schizophrenia: A Systematic Review and Meta-Analysis. Schizophr Bull. 1 mai 2017;43(3):546-56.

28. Vancampfort D, Rosenbaum S, Ward PB, Stubbs B. Exercise improves cardiorespiratory fitness in people with schizophrenia: A systematic review and meta-analysis. Schizophr Res. déc 2015;169(1-3):453-7.

29. Armstrong HF, Bartels MN, Paslavski O, Cain D, Shoval HA, Ballon JS, et al. The impact of aerobic exercise training on cardiopulmonary functioning in individuals with schizophrenia. Schizophr Res. mai 2016;173(1-2):116-7.

30. Chalfoun C, Karelis AD, Stip E, Abdel-Baki A. Running for your life: A review of physical activity and cardiovascular disease risk reduction in individuals with schizophrenia. J Sports Sci. août 2016;34(16):1500-15.

31. Hsu C-C, Liang C-S, Tai Y-M, Cheng S-L. Incongruent changes in heart rate variability and body weight after discontinuing aerobic exercise in patients with schizophrenia. Int J Psychophysiol Off J Int Organ Psychophysiol. nov 2016;109:132-7.

32. Hjorth P, Davidsen AS, Kilian R, Skrubbeltrang C. A systematic review of controlled interventions to reduce overweight and obesity in people with schizophrenia. Acta Psychiatr Scand. oct 2014;130(4):279-89.

33. Holt RIG. The prevention of diabetes and cardiovascular disease in people with schizophrenia. Acta Psychiatr Scand. août 2015;132(2):86-96.

34. Kim DD, Lang DJ, Warburton DER, Barr AM, Smith GN, Thornton AE, et al. Effects of Exercise on Serum Triglycerides and Symptoms of Schizophrenia. J Clin Psychopharmacol. avr 2017;37(2):273-4.

35. Lalande D, Thériault L, Kalinova É, Fortin A, Leone M. The effect of exercise on sleep quality and psychological, physiological, and biological correlates in patients with schizophrenia: A pilot study. Schizophr Res. mars 2016;171(1-3):235-6.

36. Costa R, Bastos T, Probst M, Seabra A, Vilhena E, Corredeira R. Sleep quality in patients with schizophrenia: The relevance of physical activity. Ment Health Phys Act. 2018;14:140-14:137.

37. Vancampfort D, Probst M, De Hert M, Soundy A, Stubbs B, Stroobants M, et al. Neurobiological effects of physical exercise in schizophrenia: a systematic review. Disabil Rehabil. 2014;36(21):1749-54. 
38. Firth J, Cotter J, Carney R, Yung AR. The Pro-Cognitive Mechanisms of Physical Exercise in People with Schizophrenia. Br J Pharmacol. 5 mars 2017;

39. Chung AKK, Chua S. Effects on prolongation of Bazett's corrected QT interval of seven second-generation antipsychotics in the treatment of schizophrenia: a meta-analysis. J Psychopharmacol Oxf Engl. mai 2011;25(5):646-66.

40. Warburton DER, Charlesworth SA, Foulds HJA, McKenzie DC, Shephard RJ, Bredin SSD. Qualified exercise professionals: best practice for work with clinical populations. Can Fam Physician Med Fam Can. juill 2013;59(7):759-61.

41. Doll-Tepper G, Dahms C, Doll B. Adapted physical activity: an interdisciplinary approach. Springer-Verlag. Berlin; 1990.

42. Stubbs B, Firth J, Berry A, Schuch FB, Rosenbaum S, Gaughran F, et al. How much physical activity do people with schizophrenia engage in? A systematic review, comparative meta-analysis and meta-regression. Schizophr Res. oct 2016;176(2-3):431-40.

43. Stubbs B, Koyanagi A, Schuch F, Firth J, Rosenbaum S, Gaughran F, et al. Physical Activity Levels and Psychosis: A Mediation Analysis of Factors Influencing Physical Activity Target Achievement Among 204186 People Across 46 Lowand Middle-Income Countries. Schizophr Bull. 24 août 2016;

44. Vancampfort D, De Hert M, Skjerven LH, Gyllensten AL, Parker A, Mulders N, et al. International Organization of Physical Therapy in Mental Health consensus on physical activity within multidisciplinary rehabilitation programmes for minimising cardio-metabolic risk in patients with schizophrenia. Disabil Rehabil. 2012;34(1):1-12.

45. Vancampfort D, Rosenbaum S, Schuch FB, Ward PB, Probst M, Stubbs B. Prevalence and predictors of treatment dropout from physical activity interventions in schizophrenia: a meta-analysis. Gen Hosp Psychiatry. avr 2016;39:15-23.

46. Firth J, Rosenbaum S, Stubbs B, Gorczynski P, Yung AR, Vancampfort D. Motivating factors and barriers towards exercise in severe mental illness: a systematic review and meta-analysis. Psychol Med. oct 2016;46(14):2869-81.

47. Yung AR, Firth J. How should physical exercise be used in schizophrenia treatment? Expert Rev Neurother. 28 déc 2016;1-2.

48. Kolt GS, Rosenkranz RR, Vandelanotte C, Caperchione CM, Maeder AJ, Tague R, et al. Using Web 2.0 applications to promote health-related physical activity: findings from the WALK 2.0 randomised controlled trial. Br J Sports Med. oct 2017;51(19):1433-40.

49. McIntosh JRD, Jay S, Hadden N, Whittaker PJ. Do E-health interventions improve physical activity in young people: a systematic review. Public Health. juill 2017;148:140-8.

50. Degroote L, Plaete J, De Bourdeaudhuij I, Verloigne M, Van Stappen V, De Meester A, et al. The Effect of the eHealth Intervention « MyPlan 1.0 » on Physical Activity in Adults Who Visit General Practice: A Quasi-Experimental Trial. Int J Environ Res Public Health. 30 janv 2018;15(2).

51. Alvarez-Jimenez M, Alcazar-Corcoles MA, González-Blanch C, Bendall S, McGorry PD, Gleeson JF. Online, social media and mobile technologies for psychosis treatment: a systematic review on novel user-led interventions. Schizophr Res. juin 2014;156(1):96-106.

52. Naslund JA, Marsch LA, McHugo GJ, Bartels SJ. Emerging mHealth and eHealth interventions for serious mental illness: a review of the literature. J Ment Health Abingdon Engl. 2015;24(5):321-32.

53. Rotondi AJ, Eack SM, Hanusa BH, Spring MB, Haas GL. Critical design elements of e-health applications for users with severe mental illness: singular focus, simple architecture, prominent contents, explicit navigation, and inclusive hyperlinks. Schizophr Bull. mars 2015;41(2):440-8.

54. Brunette MF, Rotondi AJ, Ben-Zeev D, Gottlieb JD, Mueser KT, Robinson DG, et al. Coordinated Technology-Delivered Treatment to Prevent Rehospitalization in Schizophrenia: A Novel Model of Care. Psychiatr Serv Wash DC. 1 avr 2016;67(4):444-7.

55. Gaebel W, Großimlinghaus I, Kerst A, Cohen Y, Hinsche-Böckenholt A, Johnson B, et al. European Psychiatric Association (EPA) guidance on the quality of eMental health interventions in the treatment of psychotic disorders. Eur Arch Psychiatry Clin Neurosci. mars 2016;266(2):125-37. 
56. Treisman GJ, Jayaram G, Margolis RL, Pearlson GD, Schmidt CW, Mihelish GL, et al. Perspectives on the Use of eHealth in the Management of Patients With Schizophrenia. J Nerv Ment Dis. 2016;204(8):620-9.

57. Rotondi AJ, Sinkule J, Haas GL, Spring MB, Litschge CM, Newhill CE, et al. Designing websites for persons with cognitive deficits: Design and usability of a psychoeducational intervention for persons with severe mental illness. Psychol Serv. août 2007;4(3):202-24.

58. Depp CA, Mausbach B, Granholm E, Cardenas V, Ben-Zeev D, Patterson TL, et al. Mobile interventions for severe mental illness: design and preliminary data from three approaches. J Nerv Ment Dis. oct 2010;198(10):715-21.

59. Kasckow J, Felmet K, Appelt C, Thompson R, Rotondi A, Haas G. Telepsychiatry in the assessment and treatment of schizophrenia. Clin Schizophr Relat Psychoses. avr 2014;8(1):21-27A.

60. Ricci J, Gagnon L. Evaluation du niveau d'activité physique et de condition physique. Clinic Prosport. 2011; Disponible sur:

http://www.ffsa.asso.fr/Pages/InCadres/GestClient/Communication/SportSante/Rassemblement/Evaluation_outils/testricci-gagnon_actif-inactif.pdf

61. Garber CE, Blissmer B, Deschenes MR, Franklin BA, Lamonte MJ, Lee I-M, et al. American College of Sports Medicine position stand. Quantity and quality of exercise for developing and maintaining cardiorespiratory, musculoskeletal, and neuromotor fitness in apparently healthy adults: guidance for prescribing exercise. Med Sci Sports Exerc. juill 2011;43(7):1334-59.

62. Bull FC, Maslin TS, Armstrong T. Global physical activity questionnaire (GPAQ): nine country reliability and validity study. J Phys Act Health. nov 2009;6(6):790-804.

63. Borg G. Borg's perceived exertion and pain scales. Human Kinetics. 1998. 1-120 p.

64. Wechsler D. WAIS-R manual The Psychological Corporation. New-York; 1981.

65. MEM-III; Echelle clinique de mémoire de Wechsler. 3e Edition edn. Paris; 2001. 449 p.

66. Rey A. L'examen clinique en psychologie. Paris; 1964. 1-222 p. (PUF).

67. Stroop J. Studies of interference in serial verbal reactions. Journal of Experimental Psychology. 1935;643-62.

68. Reitan R. Validity of the trail making test as an indicator of organic brain damage. Perceptual and motor skills. 1958;271-6.

69. Brickenkamp R, Zilmer E. D2 Test of attention. 1st edition edn. Göttingen, Germany; 1998. 80 p.

70. Cardebat D, Doyon B, Puel M, Goulet P, Joanette Y. [Formal and semantic lexical evocation in normal subjects. Performance and dynamics of production as a function of sex, age and educational level]. Acta Neurol Belg. 1990;90(4):207-17.

71. Kay SR, Fiszbein A, Opler LA. The positive and negative syndrome scale (PANSS) for schizophrenia. Schizophr Bull. 1987;13(2):261-76.

72. Dollfus S, Mach C, Morello R. Self-Evaluation of Negative Symptoms: A Novel Tool to Assess Negative Symptoms. Schizophr Bull. mai 2016;42(3):571-8.

73. Kirkpatrick B, Strauss GP, Nguyen L, Fischer BA, Daniel DG, Cienfuegos A, et al. The brief negative symptom scale: psychometric properties. Schizophr Bull. mars 2011;37(2):300-5.

74. Zigmond AS, Snaith RP. The hospital anxiety and depression scale. Acta Psychiatr Scand. juin 1983;67(6):361-70.

75. Guy WE. Assessment manual for Psychopharmacology. National Institute of Mental Health (Ed.). 1976;

76. Birchwood M, Smith J, Drury V, Healy J, Macmillan F, Slade M. A self-report Insight Scale for psychosis: reliability, validity and sensitivity to change. Acta Psychiatr Scand. janv 1994;89(1):62-7.

77. Denise P, Benoît O. Actisomm. Logiciel d'analyse des données actimétriques. 2016;

78. Saunders JB, Aasland OG, Babor TF, de la Fuente JR, Grant M. Development of the Alcohol Use Disorders Identification Test (AUDIT): WHO Collaborative Project on Early Detection of Persons with Harmful Alcohol Consumption-II. Addict Abingdon Engl. juin 1993;88(6):791-804.

Page $17 / 21$ 
79. Heatherton TF, Kozlowski LT, Frecker RC, Fagerström KO. The Fagerström Test for Nicotine Dependence: a revision of the Fagerström Tolerance Questionnaire. Br J Addict. sept 1991;86(9):1119-27.

80. Legleye S, Karila L, Beck F, Reynaud M. Validation of the CAST, a general population Cannabis Abuse Screening Test. Journal of Substance Use. 2007;233-42.

81. Waintraud L, Guelfi JD, Lancrenon S, Rouillon F. [Validation of M. Weissman's social adaptation questionnaire in its French version]. Ann Med Psychol (Paris). avr 1995;153(4):274-7.

82. Ryff CD, Keyes CL. The structure of psychological well-being revisited. J Pers Soc Psychol. oct 1995;69(4):719-27.

83. Kimhy D, Lauriola V, Bartels MN, Armstrong HF, Vakhrusheva J, Ballon JS, et al. Aerobic exercise for cognitive deficits in schizophrenia - The impact of frequency, duration, and fidelity with target training intensity. Schizophr Res. avr 2016;172(1-3):213-5.

84. Pajonk F-G, Wobrock T, Gruber O, Scherk H, Berner D, Kaizl I, et al. Hippocampal plasticity in response to exercise in schizophrenia. Arch Gen Psychiatry. févr 2010;67(2):133-43.

85. Semahegn A, Torpey K, Manu A, Assefa N, Tesfaye G, Ankomah A. Psychotropic medication non-adherence and associated factors among adult patients with major psychiatric disorders: a protocol for a systematic review. Syst Rev. 22 janv 2018;7(1):10.

86. Marcus BH, Ciccolo JT, Sciamanna CN. Using electronic/computer interventions to promote physical activity. Br J Sports Med. févr 2009;43(2):102-5.

87. Andersson G. Internet-Delivered Psychological Treatments. Annu Rev Clin Psychol. 2016;12:157-79.

88. Sharma A, Sharma SD, Sharma M. Mental health promotion: a narrative review of emerging trends. Curr Opin Psychiatry. sept 2017;30(5):339-45.

89. Firth J, Stubbs B, Vancampfort D, Schuch F, Lagopoulos J, Rosenbaum S, et al. Effect of aerobic exercise on hippocampal volume in humans: A systematic review and meta-analysis. Neurolmage. 1 févr 2018;166:230-166:90.

90. van der Stouwe ECD, van Busschbach JT, de Vries B, Cahn W, Aleman A, Pijnenborg GHM. Neural correlates of exercise training in individuals with schizophrenia and in healthy individuals: A systematic review. Neurolmage Clin. 2018;19:287-301.

\section{Tables}

Table 1. Inclusion and exclusion criteria for patients with schizophrenia (SZ). DSM-IV-TR: Diagnosis and Statistical Manual of Mental Disorders, 4th edition, text revision; e-APA: electronic adapted physical activity program; e-HE: electronic health education program; HE: health education program; MRI: magnetic resonance imaging. 
1. Age between 18 and 60 years old

2. Fulfill the DSM-IV-TR criteria for schizophrenia or schizoaffective disorders

3. Possibility of receiving one of the 2 interventions (e-APA or e-HE)

4. No change in psychotropic drugs during the 2 months prior to inclusion

5. Signed and informed consent

6. The need to be affiliated to a medical welfare

7. The agreement of the guardian or trustee in case of a protected major

Exclusion criteria

1. Age under 18 or over 60 years old

2. Pregnancy

3. Inclusion in another biomedical research protocol (during the present study)

4. Contraindications to MRI

5. Progressive neurological diseases

6. Physical contraindication to physical activity (moderate to severe heart failure, severe valvular disease, unstable coronary disease, acute pulmonary embolism or untreated deep venous thrombosis, uncontrolled hypertension, pulmonary arterial hypertension, treaty)

7. Neuromuscular pathologies, severe sensory and/or motor neuropathy

8. Rheumatic and articular pathologies; rheumatologic / orthopaedic problems or bone lesions at risk of fracture contraindicating physical activity

9. History of stroke or myocardial infarction less than 6 months prior to the selection visit

Table 2. Inclusion and exclusion criteria for healthy volunteers (HV). DSM-IV-TR: Diagnosis and Statistical Manual of Mental Disorders, 4th edition, text revision; e-APA: electronic adapted physical activity program; e-HE: electronic health education program; MRI: magnetic resonance imaging.

Inclusion criteria

1. Age between 18 and 60 years old

2. Possibility of receiving one of the 2 interventions (e-APA or e-HE)

3. Signed and informed consent

4. The need to be affiliated to a medical welfare

Exclusion criteria

1. Age under 18 or over 60 years old

2. Pregnancy

3. Life-long schizophrenia or schizoaffective disorder according to the DSM-IV-TR criteria

4. Inclusion in another biomedical research protocol (during the present study)

5. Contraindications to MRI

6. Progressive neurological diseases

7. Physical contraindication to physical activity (moderate to severe heart failure, severe valvular disease, unstable coronary disease, acute pulmonary embolism or untreated deep venous thrombosis, uncontrolled hypertension, pulmonary arterial hypertension, treaty)

8. Neuromuscular pathologies, severe sensory and/or motor neuropathy

9. Rheumatic and articular pathologies; rheumatologic / orthopaedic problems or bone lesions at risk of fracture contraindicating physical activity

10. History of stroke or myocardial infarction less than 6 months prior to the selection visit

Table 3. Assessments during the PEPSY V@Si trial. BMI: body mass index; BNSS: Brief Negative Syndrome Scale; B; BREQ-2: 
Behavioural Regulation Exercise Questionnaire; CGI: Clinical Global Inventory; D: day; e-APA: electronic adapted physical activity program; e-HE: electronic health education program; GPAQ: Global Physical Activity Questionnaire; HADS: Hospital Anxiety and Depression Scale; HE: health education program; IS: Insight Scale; MFI-20: Multidimensional Fatigue Inventory; MINI: MiniInternational Neuropsychiatric Interview; PANSS: Positive And Negative Syndrome Scale; RAVLT: Rey Auditory Verbal Learning Test; SNS: Self-Assessment of Negative Symptoms; TMT: Trail-Making Test; WAIS: Wechsler Adult Intelligence Scale; SAS-SR: Social Adjustment Scale-Self Report; SZ: patient with schizophrenia.

VISITS

TIME POINT (days)

\begin{tabular}{ccccccccc} 
& V1 & & V2 & V3 & V4 & V5 & V6 & V7 \\
D- & D-14 & D- & D0 & D30 & D56 & D90 & D120 & D127 \\
15 & to D0 & 7 & & & & & & \\
& & & & \pm 4 & \pm 4 & \pm 4 & $\pm 8 \mathrm{~d}$ & $\pm 2 \mathrm{~d}$ \\
& & & & $\mathrm{~d}$ & $\mathrm{~d}$ & $\mathrm{~d}$ & & \\
\hline
\end{tabular}

\section{ENROLMENT}

MINI diagnosis

Eligibility screen

$\mathrm{X}$

$\mathrm{X}$

$\mathrm{X}$

Pregnancy test

Informed consent

Sociodemographic data

Ricci and Gagnon self-questionnaire

Randomization

INTERVENTION

e-APA

e-HE

ASSESSMENTS

Concomitant treatments

Adverse events collection

Physical examinations

(blood pressure, heart rate, weight, BMI, waist circumference)

Blood analyses

(fasting glucose, triglycerides, total cholesterol, HDLc, LDLc)

Neurocognitive tests (TMT, Stroop test, WAIS Coding subtest, WAIS

Memory subtest, RAVLT, d2 test, verbal fluency, Corsi test)

Clinical self-evaluations

(HADS, SAS-SR, Ryff, Diener, ISP-25, MFI-20, BREQ-2, AUDIT, CAST,

Fagerström, Pichot, Epworth)

Clinical hetero-evaluations for SZ only

(PANSS, CGI)

Clinical evaluations for SZ only

(BNSS, SNS, IS)

Circadian typology questionnaire

GPAQ

$24 \mathrm{~h}$ body temperature

Actimetry

Sleep schedule

MRI

Exercise test and electrocardiogram

Body composition assessment

(bioelectrical impedance analysis)

Muscle strength assessment
$\mathrm{X}$

$\mathrm{X}$

$\mathrm{X}$

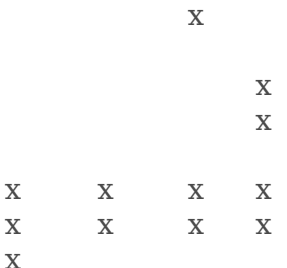

$\mathrm{X}$

$\mathrm{X}$

$\mathrm{X}$

$\mathrm{X}$

$\mathrm{X}$

$\mathrm{X}$

$\mathrm{X}$

$\begin{array}{ccc} & \mathrm{x}^{1} & \mathrm{x}^{2} \\ & \mathrm{x}^{3} & \mathrm{x}^{4} \\ & \mathrm{x}^{3} & \mathrm{x}^{4} \\ \mathrm{x} & & \\ \mathrm{x} & & \\ \mathrm{x} & & \end{array}$

$\mathrm{X}$

$\begin{array}{lll}\mathrm{X} & \mathrm{X} & \mathrm{X} \\ \mathrm{X} & \mathrm{X} & \mathrm{X} \\ \mathrm{X} & & \mathrm{x}\end{array}$

$\mathrm{X}$

$\mathrm{X}$

X

X

X

$\mathrm{X}$

$\mathrm{X}$ $\mathrm{x}^{2}$

$\mathrm{x}^{4}$ $\mathrm{x}^{4}$

(handgrip)

$\mathrm{x}^{1}$ : capsule ingestion, delivery of the temperature monitor; $\mathrm{x}^{2}$ : capsule faecal elimination, return of the temperature monitor; $\mathrm{x}^{3}$ : delivery of actimetry sensor and sleep schedule; $\mathrm{x}^{4}$ : return of the actimetry sensor and sleep schedule after one-week recording 


\section{Figures}

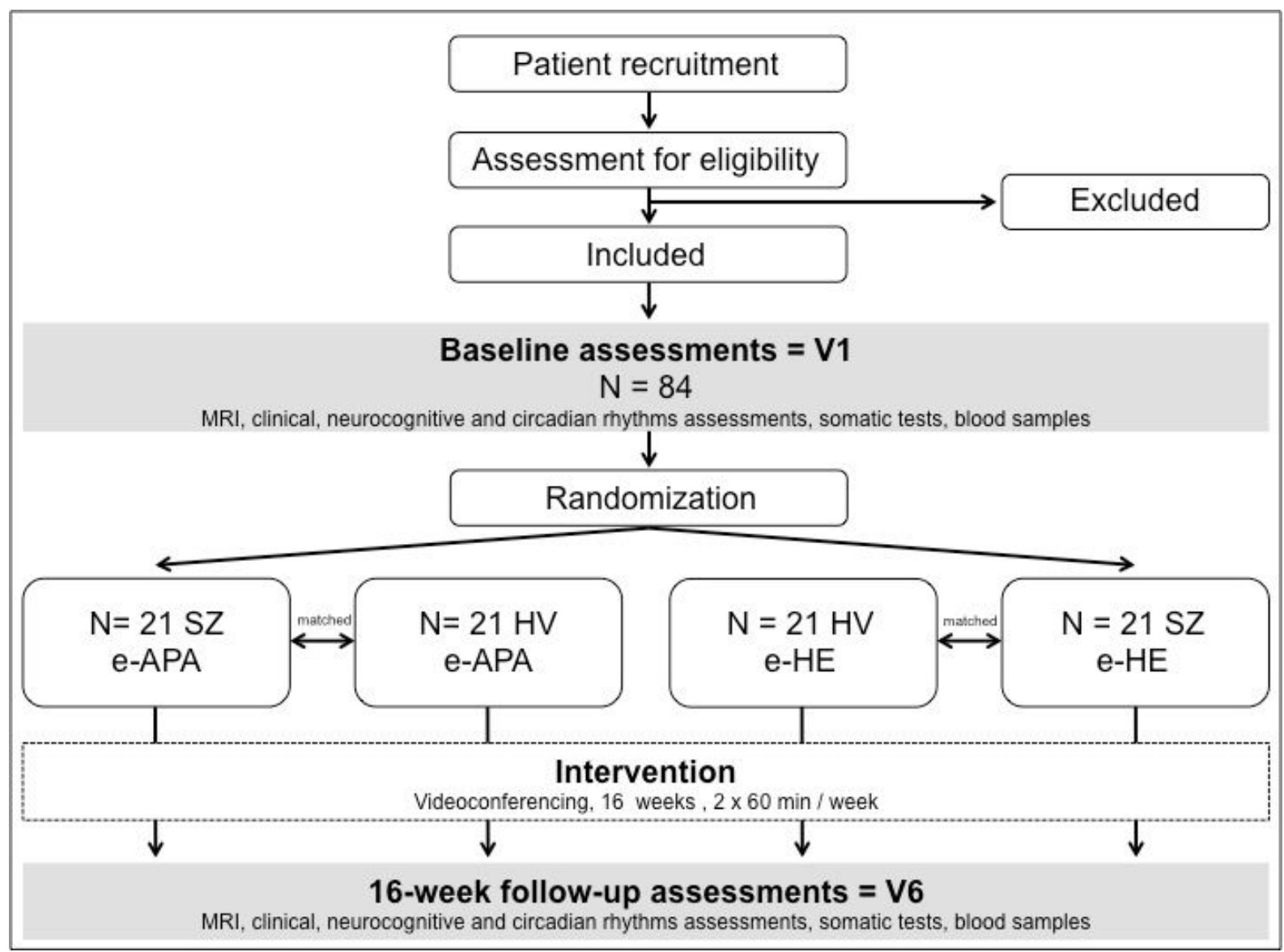

Figure 1

Participants flow chart. e-APA: electronic adapted physical activity program; e-HE: electronic health education program; HV: healthy volunteers; MRI: magnetic resonance imaging; SZ: patients with schizophrenia.

\section{Supplementary Files}

This is a list of supplementary files associated with this preprint. Click to download.

- supplement1.docx 